\title{
Intention to Take COVID-19 Vaccine and the Associations among College Students
}

\author{
Lihong Niu', Dengxin He${ }^{2}$, Xiaolan Yuan ${ }^{3}$, Minghao Pan ${ }^{4}$, Zhi Li and Qiyue Mao \\ ${ }^{1}$ Department of Respiratory and Critical Care Medicine, The Seventh Affilliated Hospital, Sun Yat-sen University, \\ Shenzhen, Guangdong Province, P.R. China \\ ${ }^{2}$ School of Nursing, Hubei University of Chinese Medicine, Wuhan, Hubei Province, P.R.China \\ ${ }^{3}$ Department of Nursing, Shandong College of Traditional Chinese Medicine, Yantai, Shandong Province, P.R.China \\ ${ }^{4}$ School of Medicine, Xinyang Normal University, Xinyang, Henan Province, P.R. China \\ ${ }^{5}$ Information Engineering College, Hubei Light Industry Technology Institute, Wuhan, Hubei Province, P.R. China
}

\begin{abstract}
Aims: This study aimed to explore factors associated with decision-making among college students on taking the coronavirus disease 2019 (COVID-19) vaccine using the Health Belief Model.

Materials and methods: This study used a web-based questionnaire to assess college students' intention to take the COVID-19 vaccine and their beliefs about COVID-19 and the vaccine. Descriptive statistics were used to characterize the sample. Independent group t-tests and one-way analysis of variance were used to assess the differences in intention to take the COVID-19 vaccine based on demographics. Pearson correlation was used to assess the associations among continuous variables. Multiple linear regression analysis was used to identify the factors associated with college students' intention to take the COVID-19 vaccine.

Results: The score for intention to take the COVID-19 vaccine was $3.76 \pm 1.12$. Scores for perceived susceptibility, perceived benefits, and perceived barriers were associated with the intention to take the vaccine.

Conclusion: Important predictors of college students' intention to take the COVID-19 vaccine included perceived high susceptibility to inflection, perceived high benefits, and perceived low barriers to receiving the vaccine. Interventions targeting these factors may be useful in facilitating acceptance of the COVID-19 vaccine.
\end{abstract}

Key words

Coronavirus disease 2019, College students, Intention to take vaccine, Health Belief Model

\section{Introduction}

The emergence of coronavirus disease 2019 (COVID-19) caused by severe acute respiratory syndrome coronavirus 2 (SARS-CoV-2) has affected humanity heavily [1]. By the end of January 2021, the World Health Organization (WHO) declared that COVID-19 directly resulted in over 2 million fatalities, and the number of confirmed cases had reached approximately 99 million across countries world wide. It is disturbing that the number of fatalities and confirmed cases continue to increase. In the absence of a COVID-19 vaccine, avoiding exposure to the virus remains the first and only line of defense. These non-pharmaceutical suppression strategies, contact tracing, limiting gatherings, restricting attendance at schools and universities, social distancing, and closing borders prevented the spread of COVID-19. These strategies resulted in the impairment of physical and psychological well-being, social interactions, and a decline in the global economy [2].
Thus, there is a great need for a safe and effective vaccine to curb the outbreaks. From a public health perspective, vaccines are recognized as the most effective strategy for dealing with outbreaks of infectious diseases [3]. There is no doubt that the COVID-19 vaccine is among the most sustainable options for managing the current pandemic. Thus far, the development of the COVID-19 vaccine has

*Corresponding author: Minghao Pan, School of Medicine, Xinyang Normal University, Xinyang, Henan Province, P.R. China

Accepted: June 18, 2021

Published online: June 21, 2021

Citation: Niu L, He D, Yuan X, et al. (2021) Intention to Take COVID-19 Vaccine and the Associations among College Students. J Healthcare 4(1):56-60

Copyright: (C) 2021 Niu L. This is an open-access article distributed under the terms of the Creative Commons Attribution License, which permits unrestricted use, distribution, and reproduction in any medium, provided the original author and source are credited. 
been relatively rapid. The safety and effectiveness of many vaccines are acceptable, and some COVID-19 vaccines are already under clinical trials [4-6]. The development of the COVID-19 vaccine warrants creating sufficient awareness to the public. College students have a strong ability to accept new things, and their intention to take the COVID-19 vaccine may influence those around them also to take the vaccine. Thus, it is reasonable to investigate college students' intention to take the COVID-19 vaccine to provide evidence for better strategies to improve acceptance of the vaccines.

The Health Beliefs Model (HBM) is widely used to understand behaviors of health and illness. This model consists of several main constructs, including perceived susceptibility, perceived severity, perceived benefits, perceived barriers, self-efficacy to engage in a behavior, and cues to action. Regarding the COVID-19 vaccine, perceived susceptibility refers to the beliefs about vulnerability to the COVID-19 infection, perceived severity refers to the beliefs about the adverse effects of the COVID-19 infection, perceived benefits refer to the beliefs about being vaccinated, and perceived barriers are the beliefs that they are restricted from being vaccinated because of psychological, physical, or financial factors. Cues to action are about information, people, and events that guide one to be vaccinated [7]. Constructs in HBM are considered essential predictors of vaccine acceptance, and it has been used in many previous studies $[8,9]$. Therefore, it is crucial to explore significant HBM constructs that are associated with college students' intention to take the COVID-19 vaccine.

\section{Materials and Methods}

\section{Study participants and survey design}

We conducted a cross-sectional survey using an online questionnaire. The researchers used the network platforms We Chat and QQ to disseminate the survey. Firstly, we sent the online questionnaire to teachers. Then, when teachers were giving a lesson to students, teachers send the online questionnaire to students. Therefore, we ensure that the respondents were only students. This study was reviewed and approved by the Xinyang Normal University Ethics Committee before the study began. The study included college students willing to participate in this study. The questionnaire was developed in Chinese. Prior to the formal survey, local experts validated the content, and the questionnaire was pilot tested.

\section{Instruments}

The questionnaire consisted of questions assessing 1) demographic characteristics, 2) HBM constructs, and 3) intention to take the COVID-19 vaccine.

\section{Demographic data}

Personal details, including gender, living area, school category, and monthly living expenses, were queried. The participants were also asked whether they or their families and friends had been diagnosed with COVID-19.

\section{Beliefs about COVID-19 and COVID-19 vaccination}

Items derived from the HBM were used to measure the students' beliefs about COVID-19 and the vaccine. The questions assessed perceived susceptibility to COVID-19 infection (four items), perceived severity of COVID-19 infection (two items), perceived benefits of a COVID-19 vaccine (two items), perceived barriers to getting a COVID-19 vaccine (four items), and cues to action (five items). Each item used a 5-point scale ("none" to "very large"). Self-efficacy was not assessed in this study because it was not necessary to understand simple health behavior. Scores $<3$ was defined as low scores, scores $\geq 3$ and $<4$ were defined as moderate scores, and scores $\geq 4$ were defined as high scores.

\section{Intention to take the COVID-19 vaccine}

The intention to take the COVID-19 vaccine was assessed using a one-item question (Overall, are you willing to take the COVID-19 vaccine?) ona 5-point scale ("none" to "very large").

\section{Statistical analysis}

SPSS software for Windows (version 20.0; IBM Corp., Armonk, NY, USA) was used for data analysis. Descriptive statistics were used to characterize the sample. Independent group $t$-tests and one-way analysis of variance were used to assess the differences in intention to take COVID-19 vaccine scores based on demographics. Pearson's correlation was used to assess associations among continuous variables. We carried out a multiple linear regression analysisto determine factors associated with the intention to take COVID-19 vaccine. Only significant factors in the $t$-tests and one-way analysis of variance, with $p$-value of $<0.05$, were selected for the multiple linear regression analysis. A $p$-value $\leq 0.05$ was considered significant.

\section{Results}

\section{Demographic data}

The survey was conducted between December 1 and December 27, 2020, and 171 complete responses were received from students with diverse demographic data. The mean score for intention to take COVID-19 vaccine was $3.76 \pm$ 1.12. As shown in (Table 1), the participants in this group had a higher representation of females (63.2\%), rural localities (69.0\%), junior college students (81.9\%), and monthly living expenses ranging $1,000-1,500$ yuan.

\section{Health beliefs}

Participants had low perceptions of susceptibility. They believed that they could partly be infected with COVID-19 because of the collective living in campus dormitories, fragile bodies, and many contacts with classmates on campus. Perceived severity was relatively high. They thought that COVID-19 was a threat to people's physical and mental health to a large extent. They believed that taking COVID-19 vaccine could be beneficial; for example, the COVID-19 vaccine may protect against the disease to a relatively large extent. Regarding barriers to taking the COVID-19 vaccine, they thought taking the vaccine may lead to minor adverse reactions. In addition, acceptance of the vaccine could be based on the following factors: Ease to access, proximity to 
Citation: Niu L, He D, Yuan X, et al. (2021) Intention to Take COVID-19 Vaccine and the Associations among College Students. J Healthcare $4(1): 56-60$

Table 1: Comparison of intention to take COVID-19 vaccine by Sample Characteristics ( $N=171)$.

\begin{tabular}{|c|c|c|c|c|}
\hline $\begin{array}{l}\text { Demographic } \\
\text { characteristics }\end{array}$ & N (\%) & $\begin{array}{l}\text { intention to take COVID-19 } \\
\text { vaccine } \\
M \pm S D\end{array}$ & $t / F$ & $\mathrm{p}$-value \\
\hline Gender & & & -1.298 & 0.169 \\
\hline Male & $63(36.8)$ & $3.90 \pm 1.19$ & & \\
\hline Female & $108(63.2)$ & $3.68 \pm 1.07$ & & \\
\hline Living area & & & 0.339 & 0.735 \\
\hline Urban & $53(31.0)$ & $3.72 \pm 1.26$ & & \\
\hline Rural & $118(69.0)$ & $3.78 \pm 1.05$ & & \\
\hline School category & & & 1.880 & 0.067 \\
\hline College & $31(18.1)$ & $3.42 \pm 1.12$ & & \\
\hline Junior college & $140(81.9)$ & $3.84 \pm 1.10$ & & \\
\hline Monthly living expenses & & & 0.488 & 0.691 \\
\hline$<1,000$ yuan & $43(25.1)$ & $3.65 \pm 1.17$ & & \\
\hline $1,000-1,500$ yuan & $110(64.3)$ & $3.77 \pm 1.10$ & & \\
\hline$>1,500$ yuan & $18(10.5)$ & $3.94 \pm 1.11$ & & \\
\hline
\end{tabular}

Table 2: Correlation between mean scores for health belief and intention to take the COVID-19 vaccine $(\mathrm{N}=171)$.

\begin{tabular}{|c|c|c|c|}
\hline \multirow[t]{2}{*}{ Health beliefs } & \multirow[t]{2}{*}{$M \pm S D$} & \multicolumn{2}{|c|}{$\begin{array}{l}\text { correlation with scores for } \\
\text { intention to take the vaccine }\end{array}$} \\
\hline & & r & p-value \\
\hline Intention to take the COVID-19 vaccine & $3.76 \pm 1.12$ & & \\
\hline \multicolumn{4}{|l|}{ Perceived susceptibility } \\
\hline Your probability of getting COVID-19 in the future & $1.94 \pm 0.95$ & 0.059 & 0.444 \\
\hline Your likelihood of getting infected due to the collective living in campus dormitories & $2.67 \pm 1.13$ & 0.174 & 0.023 \\
\hline Your likelihood of getting infected due to your fragile body & $2.67 \pm 1.10$ & 0.232 & 0.002 \\
\hline $\begin{array}{l}\text { Your possibility of getting infected based on your multiple contacts with classmates on } \\
\text { campus }\end{array}$ & $2.83 \pm 1.18$ & 0.259 & 0.001 \\
\hline \multicolumn{4}{|l|}{ Perceived severity } \\
\hline Your perception of COVID-19 as a threat to people's mental health & $4.05 \pm 0.93$ & 0.159 & 0.038 \\
\hline Your perception of COVID-19 as a threat to people's physical health & $4.43 \pm 0.83$ & 0.228 & 0.003 \\
\hline \multicolumn{4}{|l|}{ Perceived benefits } \\
\hline Your perception of the COVID-19 vaccine protecting against COVID-19 & $3.50 \pm 1.03$ & 0.488 & $<0.001$ \\
\hline Your attitude towards COVID-19 vaccine & $3.64 \pm 1.07$ & 0.265 & $<0.001$ \\
\hline \multicolumn{4}{|l|}{ Perceived barriers } \\
\hline $\begin{array}{l}\text { Your hesitation about the COVID-19 vaccine because it may lead to minor adverse } \\
\text { reactions }\end{array}$ & $2.37 \pm 0.87$ & 0.001 & 0.993 \\
\hline Your reluctance to take the COVID-19 vaccine due to adverse reactions & $1.81 \pm 0.85$ & -0.266 & $<0.001$ \\
\hline Your reluctance to take the COVID-19 vaccine because it is unsafe & $2.02 \pm 1.01$ & -0.168 & 0.028 \\
\hline $\begin{array}{l}\text { Your reluctance to take the vaccine because of the policy of the vaccine adverse } \\
\text { reaction compensation system }\end{array}$ & $2.27 \pm 1.08$ & -0.13 & 0.862 \\
\hline \multicolumn{4}{|l|}{ Cues to action } \\
\hline $\begin{array}{l}\text { How do you feel about the importance of relatives around you for the COVID-19 } \\
\text { vaccination? }\end{array}$ & $3.51 \pm 1.04$ & 0.566 & $<0.001$ \\
\hline $\begin{array}{l}\text { How do you feel about the importance of students around you for the COVID-19 } \\
\text { vaccination? }\end{array}$ & $3.59 \pm 0.99$ & 0.586 & $<0.001$ \\
\hline Will making the vaccination site close to you influence your decision on taking the vaccine? & $4.16 \pm 0.96$ & 0.185 & 0.015 \\
\hline $\begin{array}{l}\text { Will multiplying the number of vaccination sites influence your decision on taking the } \\
\text { vaccine? }\end{array}$ & $4.15 \pm 0.94$ & 0.235 & 0.002 \\
\hline Will few people at vaccination sites influence your decision on taking the vaccine? & $4.15 \pm 0.95$ & 0.256 & 0.001 \\
\hline
\end{tabular}

vaccination sites, multiplicity of vaccination sites, and few people at vaccination sites (Table 2).

\section{Intention to take COVID-19 vaccine}

Table 2 shows the correlations between scores for health beliefs and the intention to take the COVID-19 vaccine.
Table 3 shows the multiple linear regression analysis of the intention to take the COVID-19 vaccine score. In the multiple linear regression analysis, scores for the item under perceived susceptibility in the HBM, namely your possibility of getting infected based on your multiple contacts with classmates on campus, was found to be associated with scores for intention 
Citation: Niu L, He D, Yuan X, et al. (2021) Intention to Take COVID-19 Vaccine and the Associations among College Students. J Healthcare 4(1):56-60

Table 3: Multiple linear regression analysis of intention to take COVID-19 vaccine score ( $N=171)$.

\begin{tabular}{|c|c|c|c|}
\hline Variables & $\beta$ & $\mathbf{t}$ & p-value \\
\hline Constants & & 1.058 & 0.292 \\
\hline \multicolumn{4}{|l|}{ Perceived susceptibility } \\
\hline Your likelihood of getting infected due to the collective living in campus dormitories & -0.209 & -1.849 & 0.066 \\
\hline Your likelihood of getting infected due to your fragile body & 0.103 & 1.043 & 0.298 \\
\hline Your possibility of getting infected based on your multiple contacts with classmates on campus & 0.230 & 2.455 & 0.015 \\
\hline \multicolumn{4}{|l|}{ Perceived severity } \\
\hline Your perception of COVID-19 as a threat to people's mental health & -0.030 & -0.432 & 0.666 \\
\hline Your perception of COVID-19 as a threat to people's physical health & 0.091 & 1.333 & 0.184 \\
\hline \multicolumn{4}{|l|}{ Perceived benefits } \\
\hline Your perception of the COVID-19 vaccine protecting against COVID-19 & 0.167 & 2.195 & 0.030 \\
\hline Your attitude towards COVID-19 vaccine & 0.203 & 2.144 & 0.034 \\
\hline \multicolumn{4}{|l|}{ Perceived barriers } \\
\hline Your reluctance to take the COVID-19 vaccine due to adverse reactions & -0.186 & -2.056 & 0.041 \\
\hline Your reluctance to take the COVID-19 vaccine because it is unsafe & 0.025 & 0.290 & 0.772 \\
\hline \multicolumn{4}{|l|}{ Cues to action } \\
\hline How do you feel about the importance of relatives around you for the COVID-19 vaccination? & 0.159 & 1.317 & 0.190 \\
\hline How do you feel about the importance of students around you for the COVID-19 vaccination? & 0.135 & 1.029 & 0.305 \\
\hline Will making the vaccination site close to you influence your decision on taking the vaccine? & -0.022 & -0.212 & 0.832 \\
\hline Will multiplying the number of vaccination sites influence your decision on taking the vaccine? & 0.076 & 0.589 & 0.556 \\
\hline Will few people at vaccination sites influence your decision on taking the vaccine? & -0.016 & -0.135 & 0.893 \\
\hline
\end{tabular}

Note: Dependent variable: Score for intention to take COVID-19 vaccine.

$\beta=$ Standardized Beta, Adjusted $R^{2}=0.436$.

to take COVID-19 vaccine $(B=0.230, p=0.015)$. Students with higher scores for items under perceived benefits, namely your perception of the COVID-19 vaccine protecting against COVID-19 ( $B=0.167, p=0.030)$ and your attitude towards COVID-19 vaccine $(B=0.203, p=0.034)$, were found to have a higher score for intention to take COVID-19 vaccine. In addition, scores for the item under perceived barriers, namely, your reluctance to take the COVID-19 vaccine due to adverse reactions, were associated with the intention to take COVID-19 vaccine $(b=-0.186, p=0.041)$. The variables in the multiple linear regression analysis accounted for $43.6 \%$ of the variance in the score for intention to take the COVID-19 vaccine.

\section{Discussion}

This study used the HBM to examine the factors associated with the intention to take the COVID-19 vaccine among Chinese college students. Findings of the beliefs showed that college students' scores for perceived susceptibility, "probability of getting COVID-19 in the future", "your likelihood of getting infected due to the collective living in campus dormitories", and "your fragile body or multiple contacts with classmates on campus" were relatively low. The scores for perceived severity, "COVID-19 is a threat to people's mental health or physical health," were relatively high. The scores for perceived benefits, "COVID-19 vaccine can protect against COVID-19" and "attitude towards COVID-19 vaccine" were moderate. Relatively perceived low susceptibility may be due to little awareness of college students on COVID-19 because government measures have adequately controlled the COVID-19. The government's compelling explanation of COVID-19 could account for college students' relatively high perceived severity, while college students' moderate perception of benefits may be due to the moderate promotion of COVID-19 vaccines.

The overall acceptance of taking the COVID-19 vaccine was moderate. It is noteworthy that actions need to be taken on college students who have little intention of taking the COVID-19 vaccine to ensure their adherence to it and indirectly encourage their entourage to get vaccinated. The findings of this study showed that HBM constructs were associated with college students' intention to take the COVID-19 vaccine, which is consistent with many other studies $[7,10]$. Our findings suggest that perceived susceptibility, perceived benefits, and perceived barriers are important HBM constructs influencing college students' intention to take the COVID-19 vaccine, which is similar to previous study [7]. Hence, public healthintervention programsthat focus onincreasing college students' perceived susceptibility to infection and perceived benefits of the COVID-19 vaccine, whilereducing barriers of taking the COVID-19 vaccinearecommended. Findings suggest that it is not needful to promote health information framing a high risk of illness, as this has been found to be useful in influencing vaccination-related behavior [11].

This study had several limitations. First, the use of a webbased questionnaire may have led to selection bias. Second, the participants in this study were from three colleges that covered less that $1 \%$ of the whole colleges in China, limiting the generalizability of our results to all college students. Third, this study used a single item to assess the intention to take a vaccine. As the intention to take a vaccine is complex and multidimensional, it is recommended to use various types of data and measurement methods to accurately identify the intentions [12], which should be considered in future studies. 


\section{Conclusion}

College students had a moderate intention to take the COVID-19 vaccine. Significant predictors of college students' intention to take the vaccine included perceived high susceptibility to inflection, perceived high benefits, and perceived low barriers to receiving the COVID-19 vaccine. Interventions targeting these factors could be useful in promoting college students' acceptance of the COVID-19 vaccine. This study could provide reference information for the development of interventions to enhance COVID-19 vaccine acceptance.

\section{Acknowledgments}

The authors sincerely thank all the participants who willingly participated in this study.

\section{References}

1. Chakraborty I, Maity P (2020) COVID-19 outbreak: Migration, effects on society, global environment and prevention. Sci Total Environ 728: 138882.

2. Mofijur M, Fattah IMR, Alam MA, et al. (2021) Impact of COVID-19 on the social, economic, environmental and energy domains: Lessons learnt from a global pandemic. Sustainable production and consumption 26: 343-359.

3. Kennedy A, LaVail K, Nowak G, et al. (2011) Confidence about vaccines in the United States: Understanding parents' perceptions. Health Affair 30: 1151-1159.
4. Li Q, Lu H (2021) Latest updates on COVID-19 vaccines. Biosci Trends 14: 463-466.

5. Thanh Le T, Andreadakis Z, Kumar A, et al. (2020) The COVID-19 vaccine development landscape. Nat Rev Drug Discov 19: 305306.

6. Graham BS (2020) Rapid COVID-19 vaccine development. Science 368: 945-946.

7. Wong LP, Alias H, Wong PF, et al. (2020) The use of the health belief model to assess predictors of intent to receive the COVID-19 vaccine and willingness to pay. Hum Vaccin Immunother 16: 2204-2214.

8. Kan T, Zhang J (2018) Factors influencing seasonal influenza vaccination behaviour among elderly people: A systematic review. Public Health 156: 67-78.

9. Kang J, Song Y (2016) Factors associated with vaccination status among Korean submariners who are eligible for free nonmandatory influenza vaccination. Vaccine 34: 6181-6186.

10. Shahrabani S, Benzion U, Din GY (2009) Factors affecting nurses' decision to get the flu vaccine. Eur J Health Econ 10: 227-231.

11. Kim S, Pjesivac I, Jin $Y$ (2019) Effects of message framing on influenza vaccination: Understanding the role of risk disclosure, perceived vaccine efficacy, and felt ambivalence. Health Commun 34: 21-30.

12. Dube E, Gagnon D, Zhou Z, et al. (2016) Parental vaccine hesitancy in quebec (Canada). PLoS currents 8.

DOI: $10.36959 / 569 / 463$ 